\title{
ESTIMACIÓN DE PARÁMETROS GENÉTICOS PARA LA PRODUC- CIÓN DE LECHE EN LACTANCIAS DE VACAS MAMBÍ DE CUBA
}

\author{
ESTIMATION OF GENETIC PARAMETERS FOR MILK YIELD IN LACTATIONS OF \\ MAMBÍ CUBAN COWS
}

Hernández, A. ${ }^{1 \star}$, Ponce de León, R. $^{1}$, Guerra, D. ${ }^{2}$ y García, S.M. ${ }^{3}$

\begin{abstract}
${ }^{1}$ Departamento de Genética. Instituto de Ciencia Animal. San José de Las Lajas. La Habana. Cuba. *arelishdez@ica.co.cu

${ }^{2}$ Centro de Investigaciones para el Mejoramiento Animal de la Ganadería Tropical (CIMAGT). Ministerio de la Agricultura Cuba. Loma de Tierra. Cotorro. Ciudad Habana. dg@cima-minag.cu

${ }^{3}$ Empresa Pecuaria Genética de Matanzas. Finca San Andrés. Limonar. Matanzas. Cuba. epgmmtz@atenas.inf.cu
\end{abstract}

\section{Palabras clave adicionales}

Vacuno autóctono.

\section{RESUMEN}

Se utilizaron 25036 registros de producción de leche acumulada hasta 305 días (13 495 de primera lactancia (L1), 6067 de la segunda (L2), 3445 de la tercera (L3) y 2029 de la cuarta (L4), respectivamente) pertenecientes a 13495 vacas Mambí de Cuba (3/4 Holstein 1/4 Cebú) que parieron entre los años 1981-2006, distribuidas en 173 rebaños de 4 empresas ganaderas. El objetivo de este estudio fue estimar las heredabilidades $y$ correlaciones genéticas entre las cuatro primeras lactancias (L1-L4) y determinar si la primera lactancia podría ser considerada como criterio de selección de vacas y sementales de esta raza. Para estimar los componentes de (co) varianza y parámetros genéticos se utilizó el programa REMLF90 empleando un modelo animal multivariado que incluyó como efecto fijo el grupo de contemporáneos (rebaño-año-cuatrimestre de parto), la edad al parto como covariable lineal y cuadrática, y como efectos aleatorios el animal y el error. Los promedios para L1 hasta L4 fueron de: 1957 kg, 2086 kg, 2099 kg y 2106 kg, y los valores de heredabilidad fueron $0,22 \pm 0,03 ; 0,17 \pm 0,04$; $0,22 \pm 0,05$ y $0,26 \pm 0,06$, respectivamente. Las correlaciones genéticas entre las cuatro primeras lactancias fueron altas con valores entre 0,76 y 0,95 . Se concluye que es posible obtener ganancias genéticas mediante selección por producción de leche; que hubo un alto porcentaje de genes en

\author{
AdDiTIONAL KEYWORDS \\ Local cattle.
}

común que influyeron en las diferentes lactancias, y que la producción lechera en la primera lactancia es un buen indicador del comportamiento productivo de las vacas Mambí de Cuba, que puede ser utilizada como criterio de selección.

\section{SUMMARY}

The records of 25036 lactations of milk yield up to 305 days (13 495 in first lactation (L1), 6067 in the second (L2), 3445 in the third (L3) and 2029 in the fourth (L4), respectively) from 13495 Cuban Mambí ( $3 / 4$ Holstein $1 / 4$ Cebú) cows were used. The cows calved between 1981 and 2006 in 173 herds of four genetic farms. The goal of this study was to estimate the heritability and genetic correlations the first four lactations (L1-L4) and determine if the first lactation could be considered as selection criteria for cows and sires of the breed. The REMLF90 program was used for estimating the (co) variance components and the genetic parameters by means of a multivariate animal model that included the contemporary group (herd-yearcalving season) as fixed effect, calving age as linear and quadratic covariable, and the animal and the error as random effects. The means for L1 up to L4 were of $1957 \mathrm{~kg}, 2086 \mathrm{~kg}, 2099 \mathrm{~kg}$ and $2106 \mathrm{~kg}$, and the heritability values were of 0.22 $\pm 0.03,0.17 \pm 0.04,0.22 \pm 0.05$ and $0.26 \pm 0.06$, 
respectively. The genetic correlations between the first four lactations were high, with values between 0.76 and 0.95 . It was concluded that it is possible to obtain genetic gain through milk yield selection, that a common group of genes are affecting the different lactations, and that milk yield in the first lactation is a good indicator for productive performance of Cuban Mambí cows, that can be used as selection criteria.

\section{INTRODUCCIÓN}

Los resultados obtenidos por varios investigadores como Albuquerque et al. (1999) concuerdan en que el comportamiento animal durante varias lactancias está influenciado por más o menos los mismos genes, con un mínimo de variación, y por lo tanto, la producción obtenida en la primera lactancia podía ser un criterio de selección eficiente. Meyer (1984) planteó que cuando las lactancias posteriores eran incluidas en la estimación del valor genético se creaban más conexiones entre sementales permitiendo incrementar la exactitud de las evaluaciones. Otros autores como Teepker y Swalve (1988), Alburquerque et al. (1996), Pösö y Mäntysaary (1996) y Guo et al. (2002) expresaron que el hecho de incluir más lactancias podía causar un cambio en el orden del valor genético debido a los efectos de la selección.

Márquez et al. (2003) comentaron acerca de las ventajas y desventajas de utilizar sólamente los registros de la primera lactancia en la evaluación de los sementales. Dentro de las ventajas mencionó la existencia de un sesgo mínimo de la selección de las hijas, disminución del efecto de los factores de ajuste por edad y la reducción de los requerimientos computacionales; y como desventajas la reducción en el número de hijas contemporáneas, la pérdida de relaciones fenotípicas entre la primera lactancia y la producción en toda la vida y las posibles diferencias genéticas entre lactancias.

Se han realizado varios estudios relacionados con la estimación de parámetros genéticos de la producción lechera en diferen- tes lactancias, ya sea considerando las tres primeras lactancias (Meyer, 1984; Swalve y Van Vleck, 1987; Raheja et al.,1989; Visscher y Thompson, 1992; Albuquerque et al., 1996; Albuquerque et al., 1999; Freitas et al., 2001; Márquez et al., 2003; Carlén et al., 2004; Strabel y Jamrozik, 2006) o las cuatro primeras lactancias (García-Cortés et al., 1995; Ribas et al., 2001; Palacios-Espinoza et al., 2007). En general, la mayoría de estos estudios fueron en razas especializadas en la producción lechera.

El programa de cruzamientos para la producción de leche en Cuba se desarrolló desde la década del 60 y se sustentó en la utilización del Cebú (95\% de la población bovina en ese momento) y del Holstein como raza mejoradora (López y Ribas, 1993). Para la formación de la raza Mambí de Cuba se utilizó semen de toros Holstein importados de Canadá, de alto valor genético, quienes también fueron usados en una segunda generación sobre hembras F1. Se realizaron apareamientos inter-se de los $3 / 4$ Holstein $1 / 4$ Cebú y posteriormente entre sus descendientes, y con apoyo de la selección por prueba de progenie para machos y la elección de vacas élites como madres de sementales se obtuvo la nueva raza. La estabilización de este nivel de cruzamiento perseguía lograr un genotipo adaptado a las condiciones tropicales que manifestara un alto potencial productivo y que mantuviera las características deseables de rusticidad y resistencia del Cebú.

El objetivo del presente trabajo fue estimar las heredabilidades y las correlaciones genéticas y ambientales de la producción de leche acumulada hasta los 305 días, en las cuatro primeras lactancias de vacas Mambí de Cuba, con el propósito de determinar si la primera lactancia podía ser considerada como criterio de selección de las vacas y los sementales de esta raza.

\section{MATERIAL Y MÉTODOS}

Se utilizaron inicialmente 50079 regis- 
tros de la producción de leche acumulada hasta los 305 días (L305) de vacas Mambí de Cuba (3/4 Holstein $1 / 4$ Cebú), procedentes de 4 ganaderías (Empresa Pecuaria Genética de Matanzas, Empresa Bacuranao, Empresa Los Naranjos y Empresa Camilo Cienfuegos). Todas estaban localizadas en la región occidental de la República de Cuba, situada en el golfo de México, entre 20 a $23^{\circ}$ Ny 74 a $85^{\circ}$ O. Según Anónimo (2009) en la mayor parte de Cuba el clima predominante es de tipo cálido tropical con dos estaciones: la lluviosa, comprendida entre los meses de mayo a octubre, y la seca de noviembre a abril. La temperatura media anual es de $24^{\circ} \mathrm{C}$, la humedad relativa media presenta promedios cercanos al $80 \%$ y las precipitaciones superan los $3000 \mathrm{~mm}$ al año.

Solo se utilizaron los registros de aquellos animales que tenían información de su primera lactancia. Para garantizar una mayor precisión en la estimación de los parámetros, se eliminaron los datos que presentaban baja frecuencia en: edad al parto inferior a los 24 meses ( $0,08 \%$ de los datos), producción de leche por día inferior a $1 \mathrm{~kg}(0,72 \%)$ y lactancias con menos de 30 días de duración (0,56\%). También se eliminaron los valores por encima y por debajo de 3 desviaciones estándar de la media y los grupos de contemporáneos conformados por menos de 3 animales, previendo que las hembras fueran hijas de diferentes sementales. Se consideró como grupos de contemporáneos la combinación de rebaño-año-cuatrimestre de parto. En esta raza se utiliza la inseminación artificial y los sementales tienen hijas distribuidas en las cuatro ganaderías estudiadas.

El fichero depurado presentó un total de 25036 lactancias: 13495 de primera lactancia (L1), 6067 de segunda (L2), 3445 de tercera (L3) y 2029 de cuarta lactancia (L4), respectivamente; pertenecientes a 13495 vacas que parieron entre los años 1981 y el 2006 y se distribuyeron en 173 rebaños. Estas vacas fueron hijas de 5581 madres y 242 padres.
El sistema de alimentación fue basado en pastoreo. Las vacas lactantes consumieron fundamentalmente pasto estrella (Cynodon nlemfuensis), pasto pangola (Digitaria decumbens), pasto guinea (Panicum maximum) y algunas especies de pastos naturales. Después del año 2000 se comenzó la introducción del pasto king grass (Pennisetum purpureum var. Cuba CT-115), con destino al pastoreo y como reserva estratégica de los períodos de sequía.

Los criterios prevalecientes en los años de trabajo respecto a la suplementación se pueden indicar de la forma siguiente:

1981-1990. Recibieron diariamente alimento concentrado comercial de forma individual a razón de 0,46 kg/litro de concentrado después del quinto litro de leche producido.

1991-2000. Hubo una baja muy considerable en rotación de potreros y suplementación casi cero, promediando apenas $1 \mathrm{~kg} /$ animal.

2001-2006. Se logró mejorar el acuartonamiento y se comenzó a ofrecer Norgold (granos secos de destilería con solubles, subproducto resultante del proceso de producción de etanol a partir del maíz) a razón de $0,46 \mathrm{~kg} / \mathrm{litro}$ desde el segundo litro producido, aproximadamente.

Durante el período de seca fue suministrado suplementación de caña de azúcar (Saccharum officinarum), king grass (Pennisetum purpureum) en forma de forraje y hollejo de cítrico, según disponibilidad. La urea y las sales minerales formaban parte de los alimentos complementarios según lo requería la dieta.

Las hembras no lactantes se mantuvieron en las mismas condiciones de pastoreo. A los treinta días preparto recibieron suplementación con concentrado, según disponibilidad, no sobrepasando de 2-3 kg/vaca/ día. Durante el período de 1991 al 2000, no se ofreció ningún suplemento a las vacas preparto. Se realizó ordeño mecánico 2 veces al día. Los intervalos entre ordeños 
fueron de 10 y 14 horas. Los horarios de pastoreo en las principales empresas estudiadas fueron los denominados normales (mañana, tarde y noche).

Para estimar los componentes de (co) varianza y los parámetros genéticos (heredabilidades y correlaciones) se empleó el programa REMLF90 (Misztal, 1999), el cual utiliza el procedimiento REML. Se utilizó un modelo animal multivariado para estimar la heredabilidad en cada una de las lactancias, considerando cada lactancia como una característica independiente. También se estimaron las correlaciones genéticas y ambientales entre lactancias. El modelo utilizado fue:

$$
\begin{gathered}
\mathrm{y}_{\mathrm{i}}=\mathrm{X}_{\mathrm{i}} \mathrm{b}_{\mathrm{i}}+\mathrm{Z}_{\mathrm{i}} \mathrm{a}_{\mathrm{i}}+\mathrm{e}_{\mathrm{i}} \\
{\left[\begin{array}{l}
y_{1} \\
y_{2} \\
y_{3} \\
y_{4}
\end{array}\right]=\left[\begin{array}{cccc}
X_{1} & 0 & 0 & 0 \\
0 & X_{2} & 0 & 0 \\
0 & 0 & X_{3} & 0 \\
0 & 0 & 0 & X_{4}
\end{array}\right]\left[\begin{array}{l}
b_{1} \\
b_{2} \\
b_{3} \\
b_{4}
\end{array}\right]} \\
+\left[\begin{array}{cccc}
Z_{1} & 0 & 0 & 0 \\
0 & Z_{2} & 0 & 0 \\
0 & 0 & Z_{3} & 0 \\
0 & 0 & 0 & Z_{4}
\end{array}\right]\left[\begin{array}{l}
a_{1} \\
a_{2} \\
a_{3} \\
a_{4}
\end{array}\right]+\left[\begin{array}{l}
e_{1} \\
e_{2} \\
e_{3} \\
e_{4}
\end{array}\right]
\end{gathered}
$$

donde:

$\mathrm{y}_{\mathrm{i}}$ : vector de observaciones para la i-ésima lactancia;

$b_{i}$ : vector de efectos fijos para la i-ésima lactancia, donde se incluyó el efecto del grupo de contemporáneo (rebaño-año-cuatrimestre de parto) y la edad al parto como covariable lineal y cuadrática;

$a_{i}$ : vector de efectos genéticos aditivos para la iésima lactancia;

$\mathrm{e}_{\mathrm{i}}$ : vector aleatorio (error) para la i-ésima lactancia;

$\mathrm{X}_{\mathrm{i}}$ : matriz de incidencia de efectos fijos;

$Z_{\mathrm{i}}$ : matriz incidencia de efectos aleatorios.

\section{RESULTADOSYDISCUSIÓN}

La descripción estadística de los registros de la producción de leche hasta 305 días, de la primera a la cuarta lactancia se presenta en la tabla I. El número de registros empleados para el estudio de cada lactancia decreció en un 55,0\%, 43,2\% y 74,5\% de la primera a la segunda lactancia, de la segunda a la tercera lactancia y de la primera a la tercera lactancia, respectivamente. Por lo tanto, sólo el 25,5\% de los registros estuvieron disponibles al finalizar la tercera lactancia. Un comportamiento similar de los resultados fue observado por Albuquerque et al. (1999).

Los promedios de la producción de leche de la primera a la cuarta lactancia en las vacas Mambí de Cuba estuvieron entre 1957 \pm 7 y $2106 \pm 19 \mathrm{~kg}$. Estos resultados fueron inferiores a aquellos obtenidos bajo condiciones tropicales en las tres primeras lactancias de vacas Gir (2204 \pm 289, $2699 \pm$ 208 y $2691 \pm 122$ kg) y Holstein (5521 \pm 1523 , $6421 \pm 1789$ y $7045 \pm 1903 \mathrm{~kg}$ ) en Brasil, por Albuquerque et al. (1999) y Freitas et al. (2001), y a los resultados de Márquez et al. (2003) en dos rebaños de vacas Holstein en Baja California, México con $8146 \pm 1310$ kg y $8246 \pm 1324 \mathrm{~kg}$ en la primera lactancia, 8896 $\pm 1125 \mathrm{~kg}$ y $8690 \pm 1275 \mathrm{~kg}$ en la segunda

Tabla I. Número de observaciones (N), media, desviación estándar (DE) y coeficiente de variación $(\mathrm{CV})$ para la producción de leche, de las primeras cuatro lactancias (L1L4) de vacas Mambí de Cuba. (Number of observations (N), mean, standard deviation (DE) and coefficient of variation (CV) of milk yield, for the first four lactations (L1- L4) of Cuban Mambí cows).

\begin{tabular}{lcccc}
\hline & $\mathrm{N}$ & Media $(\mathrm{kg})$ & DE & CV (\%) \\
\hline L1 & 13495 & 1957 & 784 & 40 \\
L2 & 6067 & 2086 & 927 & 44 \\
L3 & 3445 & 2099 & 975 & 44 \\
L4 & 2029 & 2106 & 977 & 46 \\
\hline
\end{tabular}


lactancia y $9239 \pm 1173 \mathrm{~kg}$ y $9522 \pm 1142 \mathrm{~kg}$ en la tercera lactancia, para los dos rebaños respectivamente. Bajo las condiciones ambientales cubanas, se alcanzaron promedios similares en el Holstein (2260 \pm 789 , $2204 \pm 815,2274 \pm 752$ y $2355 \pm 760 \mathrm{~kg})$ citados por Palacios-Espinoza et al. (2007).

Los promedios de la producción de leche aumentaron de la primera a la cuarta lactancia donde presentó su mayor valor $(2106 \pm 19 \mathrm{~kg})$. En estudios previos realizados en esta raza utilizando todas las lactancias (Hernández et al., 2005) obtuvieron los mayores promedios de la cuarta a la séptima lactancia. Palacios-Espinoza et al. (2007) en vacas Holstein en Cuba, también obtuvieron mayores promedios en la cuarta lactancia (2355 $\pm 760 \mathrm{~kg})$.

En la tabla II aparecen los estimados de los componentes de varianza genética aditiva $\left(\sigma^{2} a\right)$, varianza residual $\left(\sigma^{2} e\right)$, varianza fenotípica $\left(\sigma^{2} f\right)$ y la heredabilidad $\left(h^{2}\right)$, en cada una de las lactancias estudiadas. En la varianza genética aditiva se observó un incremento de la primera a la cuarta lactancia, similar a lo obtenido por Freitas et al. (2001).

El menor valor para la varianza residual se obtuvo en la primera lactancia e incrementó su valor en las lactancias posterio-

Tabla II. Estimadores de la varianza genética aditiva $\left(\sigma^{2} a\right)$, varianza residual $\left(\sigma^{2} e\right)$, varianza fenotípica $\left(\sigma^{2} f\right)$ y heredabilidad $\left(h^{2}\right)$ para la producción de leche, de las primeras cuatro lactancias (L1-L4) de vacas Mambí de Cuba. (Estimators of additive genetic variance $\left(\sigma^{2} \mathrm{a}\right)$, residual variance $\left(\sigma^{2} \mathrm{e}\right)$, phenotypic variance $\left(\sigma^{2 f}\right)$ and heredability $\left(h^{2}\right)$ of milk yield, for the first four lactations (L1-L4) of Cuban Mambí cows).

\begin{tabular}{ccccc}
\hline & $\sigma^{2} \mathrm{a}$ & $\sigma^{2} \mathrm{e}$ & $\sigma^{2 f}$ & $\mathrm{~h}^{2} \pm \mathrm{EE}$ \\
\hline L1 & 76200 & 266100 & 342300 & $0,22 \pm 0,03$ \\
L2 & 79760 & 380600 & 460360 & $0,17 \pm 0,04$ \\
L3 & 112200 & 390000 & 502200 & $0,22 \pm 0,05$ \\
L4 & 125300 & 348500 & 473800 & $0,26 \pm 0,06$ \\
\hline
\end{tabular}

res. Resultados similares a los Albuquerque et al. (1999), Freitas et al. (2001) y a los de Palacios-Espinoza et al. (2007). Valente et al. (1995) y Teixeira et al. (1996) señalaron que este comportamiento podía deberse a factores que afectaron el comportamiento reproductivo del animal, como el período seco previo al parto, el período de servicio, entre otros que no influyeron en la primera lactancia, pero si en las siguientes. La varianza fenotípica mostró igual comportamiento que la varianza residual.

La heredabilidad para la producción de leche en la primera lactancia $(0,22)$ fue superior a los 0,15 obtenidos en el Siboney de Cuba mediante un modelo animal univariado (González-Peña, 2006) y a los 0,17 en el Holstein en Cuba, también empleando un modelo animal multivariado (Palacios-Espinosa et al., 2007).

Se apreciaron estimados de heredabilidades entre 0,17 y 0,26 en las cuatro primeras lactancias. Los valores obtenidos en las tres primeras lactancias $(0,17$ a 0,22$)$ se corresponden con los resultados de Raheja et al. (1989) en vacas Holstein Canadienses mediante un modelo de máxima verosimilitud $(0,18,0,18$ y 0,19$)$. También coincide con los resultados alcanzados con el empleo del modelo animal, entre ellos los de Albuquerque et al. (1999) en el Gir (0,20, 0,12 y 0,19), los de Freitas et al. (2001) en el Holstein en Brasil con valores de 0,16 $\pm 0,09$; $0,17 \pm 0,11$ y $0,16 \pm 0,10$ y los de PalaciosEspinoza et al. (2007) con 0,17; 0,16; 0,16 y 0,14 , respectivamente. Otros trabajos que presentaron similitud fueron los de Ribas et al. (2001) en el Siboney de Cuba (0,18 $\pm 0,05$; $0,11 \pm 0,04 ; 0,13 \pm 0,07$ y $0,12 \pm 0,05$ en primer, segundo, tercer y cuarto o más partos) mediante un modelo padre y los de Strabel y Jamrozik (2006) en vacas blancas y negras polacas quiénes obtuvieron estimados de 0,$18 ; 0,16$, and 0,17 usando un modelo del día de control con regresiones aleatorias.

Otros autores informaron mayores estimados de heredabilidades en las tres primeras lactancias de vacas Holstein $(0,23$ a 0,40$)$ 
mediante el uso del modelo animal (Albuquerque et al., 1996; Swalve y Van Vleck, 1987; Visscher y Thompson, 1992; Carlén et al., 2004).

Las correlaciones genéticas y ambientales entre lactancias se muestran en la tabla III. Las correlaciones genéticas entre las cuatro primeras lactancias presentaron valores entre 0,76 y 0,95, evidenciando que hubo un alto porcentaje de genes en común que influyeron en las diferentes lactancias. Las correlaciones ambientales (entre 0,14 y 0,43 ) fueron inferiores a las correlaciones genéticas y están en el rango de lo informado por Carlén et al. (2004).

En el Mambí de Cuba la mayor correlación genética se alcanzó entre la primera y la segunda lactancia $(0,95)$, lo cual no se corresponde con lo obtenido en el Holstein donde las menores correlaciones genéticas se obtuvieron entre la primera y las demás lactancias (Meyer, 1984; Swalve y VanVleck, 1987; Albuquerque et al., 1996; Carlén et al., 2004; Palacios-Espinosa et al., 2007). Estos resultados contradictorios pueden deberse a la alta presión de selección que se realiza en la primera lactancia en la raza Holstein, no sucediendo así en el Mambí de Cuba donde se ha efectuado poca selección en primera lactancia al no disponer de suficientes novillas de reemplazo.

Las tres primeras lactancias son las de

Tabla III. Correlaciones genéticas (arriba de la diagonal) y ambientales (debajo de la diagonal) para la producción de leche entre lactancias (L1 a L4) de vacas Mambí de Cuba. (Genetic (above the diagonal) and environment (below the diagonal) correlation of milk yield among lactations (L1 to L4) of Cuban Mambí cows).

\begin{tabular}{lcccc}
\hline & L1 & L2 & L3 & L4 \\
\hline L1 & - & $0,95 \pm 0,05$ & $0,83 \pm 0,08$ & $0,76 \pm 0,10$ \\
L2 & $0,37 \pm 0,02$ & - & $0,89 \pm 0,07$ & $0,82 \pm 0,11$ \\
L3 & $0,32 \pm 0,03$ & $0,40 \pm 0,03$ & - & $0,76 \pm 0,13$ \\
L4 & $0,14 \pm 0,04$ & $0,25 \pm 0,05$ & $0,43 \pm 0,04$ & - \\
\hline
\end{tabular}

mayor interés por ser las que se obtienen más tempranamente y por aportar la mayor cantidad de datos. En el presente trabajo representan el 75,5\% de la información lechera. Estas lactancias presentaron altas correlaciones genéticas entre sí $(0,83$ - 0,95) por lo que se puede considerar que la primera lactancia es un buen indicador del comportamiento productivo de las vacas Mambí de Cuba y puede ser utilizada como criterio de selección basado en lo informado por Robertson (1959) quién sugirió que para propósitos de selección debían considerarse correlaciones genéticas superiores a 0,80.

Los estimados de las correlaciones genéticas entre las tres primeras lactancias coinciden con los resultados de Swalve y Van Vleck (1987), Visscher y Thompson (1992), Albuquerque et al.(1996), Freitas et al. (2001), Carlén et al. (2004) y Yang et al . (2005) quiénes obtuvieron valores entre 0,81 y 1,00 .

Otros autores, encontraron correlaciones genéticas entre las tres primeras lactancias inferiores a los valores anteriores, entre ellos, Márquez et al. (2003) obtuvieron valores de 0,79 a 0,84 , PalaciosEspinoza et al. (2007) entre 0,62 y 0,73 y Zavertyaev y Prozherin (2008) de 0,40 a 0,53 , respectivamente.

\section{CONCLUSIONES}

Los resultados obtenidos en el presente trabajo se corresponden con lo informado por diversos autores en otras razas lecheras.

Los estimados de heredebilidad entre lactancias fueron de medianos a bajos, indicando la posibilidad de obtener ganancias genéticas mediante la selección por producción de leche. Los resultados obtenidos en las correlaciones genéticas entre las cuatro primeras lactancias evidenciaron que hubo un alto porcentaje de genes en común que influyeron en las diferentes lactancias. Las altas correlaciones genéticas de la primera lactancia con el resto de las lactancias

Archivos de zootecnia vol. 60, núm. 232, p. 856. 


\section{PARÁMETROS GENÉTICOS PARA PRODUCCIÓN LÁCTEA EN VACAS MAMBÍ CUBANAS}

demostraron que la primera lactancia es un buen indicador del comportamiento productivo de las vacas Mambí de Cuba y puede ser utilizada como criterio de selección de las vacas y los sementales de esta raza.

\section{BIBLIOGRAFÍA}

Albuquerque, L.G., Keow, J.F. and Van Vleck, L.D. 1996. Genetic parameters of milk, fat and protein yields in the first three lactations, using an animal model and restricted maximum likeli-hood. Rev. Bras. Genet., 19: 79-86.

Albuquerque, M.SM., Freitas, M.A.R. and Teodoro, R.L. 1999. Genetic and phenotypic parameters of productivity traits on the first three lactations in Gyr cattle herds. Genet. Mol. Biol., 22: 177181.

Anónimo. 2009. El clima en Cuba. http://www. bedincuba.com/2climaencuba.htm (07/02/09).

Carlén, E., Strandberg, E. and Roth, A. 2004. Genetic parameters for clinical mastitis, somatic cell score, and production in the first three lactations of Swedish Holstein cows. J. Dairy Sci., 87: 3062-3070.

Freitas, A.F., Durães, M.C. e Valente, J. 2001. Parâmetros genéticos para produção de leite e gordura nas três primeiras lactações de vacas Holandesas. Rev. Bras. Zootecn., 30: 709-713.

García-Cortés, L.A., Moreno, L. and Varona, L. 1995. (Co) variance components estimation of yield traits between different lactations using animal model. Livest. Prod. Sci., 43: 111-117.

González-Peña, D. 2006. Evaluación genética del ganado Siboney de Cuba empleando la producción del día de control bajo un modelo de regresión aleatoria. Tesis (Doctor en Ciencias). Centro de Investigaciones para el Mejoramiento Animal. Ciudad de La Habana. 118 pp.

Guo, Z., Lund, M.S., Madsen, P., Korsgaard, I. and Jensen, J. 2002. Genetic parameters estimation for milk yield over multiple parities and various lengths of lactation in Danish Jerseys by random regression models. J. Dairy Sci., 85: 1596-1606.

Hernández, A., Ponce de León, R., Gutiérrez, M., García, R., García, S.M, Mora, M. y Guzmán, G. 2005. Efectos ambientales en la producción lechera de la raza bovina Mambí de Cuba. Rev.

\section{AGRADECIMIENTOS}

Se agradece a los trabajadores y responsables de las unidades de producción por su cooperación para el desarrollo del presente trabajo.

Cuba. Cienc. Agric., 39: 533-542.

López, D. y Ribas, M. 1993. Formación de nuevas razas lecheras. Resultados en Cuba. Rev. Cuba. Cienc. Agric. 27: 1.

Márquez, A.P., Correa, A. and Cobos, S. 2003. Estimates of genetic parameters for milk yield of Holstein cows in two dairy herds in Baja California, Mexico. Proceedings Western Section American Society of Animal Science. 54: 1-3.

Meyer, K. 1984. Estimates of genetic parameters for milk and fat yield for the first three lactations in British Fresian cows. Anim. Prod., 38: 313322.

Misztal, I. 1999. REMLF90 Manual. http://nce. ads.uga.edu/ ignacy/numpub/blupf90/docs/ remlf90.pdf (02/03/08).

Palacios-Espinoza, A., Espinoza Villavicencio, J. L., González-Peña, D., Guerra Iglesias, D., de la Peña, R.L. and Rodríguez Almeida, F. 2007. Estimation of covariance components for the first four lactations in Holstein cattle according to different models. Zootec. Trop., 25: 9-18.

Pösö, J. and Mäntysaari, E.A. 1996. Genetic relationships between reproductive disorders, operational days open and milk yield. Livest. Prod. Sci., 46: 41-48.

Raheja, K.L., Burnside, E.B. and Schaeffer, L.R. 1989. Relationships between fertility and production in Holstein dairy cattle in different lactations. J. Dairy Sci., 72: 2670-2678.

Ribas, M., Ponce de León, R., Ajete, A., Mederos, R.E., Gutiérrez, M. y Sosa, E. 2001. Mejoramiento genético de la producción de leche bovina bajo las condiciones actuales de producción. Informe final de proyecto. ICA. La Habana. Cuba. 71 pp.

Robertson, A. 1959. The sampling variance of the genetic correlation coefficient. Biometrics, 15: 469-485.

Strabel, T. and Jamrozik, J. 2006. Genetic analysis 


\section{HERNÁNDEZ, PONCE DE LEÓN, GUERRA Y GARCÍA}

of milk production traits of Polish Black and White cattle using large-scale random regression test-day models. J. Dairy Sci., 89: 3152-3163.

Swalve, H. and Van Vleck, L.D. 1987. Estimation of genetic (co) variances for milk yield in first three lactations using an animal model and restricted maximum likelihood. J. Dairy Sci., 70: 842-849.

Teepker, G. and Swalve, H.H. 1988. Estimation of genetic parameters for milk production in the first three lactations. Livest. Prod. Sci., 20: 193-202.

Teixeira, N.M., Valente, J. e Verneque, R.S. 1996. Factores de ajustamento da produção de leite para período de serviço e período seco na raçã Holandesa. Anais Reunião Anual da Sociedade Brasileira de Zootecnia. SBZ. Fortaleza. pp. 54-56.

Valente, J., Teixeira, N.M. e Verneque, R.S. 1995. Efeitos dos períodos de serviço anterior, período seco anterior e período de serviço corrente sobre a produção de leite. Reunião Anual da Sociedade Brasileira de Zootecnia, 32. Brasília. Anais. SBZ. Brasília. pp. 686-688.

Visscher, P.M and Thompson, R. 1992. Univariate and multivariate parameter estimates for milk production traits using an animal model. I. Description and results of REML analyses. Genet. Sel. Evol., 24: 415-429.

Yang, R.Q., Ren, H.Y., Schaeffer, L.R. and Xu, S.Z. 2005.Estimation of genetic parameters for lactational milk yields using two-dimensional random regressions on parities and days in milk in Chinese Simmental cattle. J. Anim. Breed. Genet., 122: 49-55.

Zavertyaev, B.P. and Prozherin, V.P. 2008. Estimation and use of repeatability and heritability coefficients of productivity traits in breeding dairy cattle. Russian Agric. Sci., 34: 111-113. http://www.springerlink.com/content/ I697365nq1774440 (15/05/09). 